\title{
Electron Injection into Fermi Acceleration in Quasiparallel Collisionless Shocks: Combining Hybrid Simulations with Test Particle Acceleration
}

\author{
Adrian Hanusch* \\ Rostock University, Germany \\ E-mail: adrian.hanuscheuni-rostock.de \\ Tatyana Liseykina \\ Rostock University, Germany \\ E-mail: tatyana.liseykina@uni-rostock.de

\section{Mikhail Malkov} \\ University of California, San Diego, USA \\ E-mail: mmalkov@ucsd.edu
}

\begin{abstract}
In situ observations of energetic particles at the Earth's bow-shock have long created an opinion that electrons are most efficiently accelerated (injected) in a quasi-perpendicular, while protons in quasi-parallel shock geometry. Shocks that deemed responsible for the production of cosmic ray electrons and their radiation from sources such as supernova remnants are, however, much more powerful and larger. Their remote observations suggest that electrons are accelerated very efficiently in the quasi-parallel geometry. We investigate the possibility that protons accelerated to high energies create sufficient wave turbulence required for the electron injection into the diffusive shock acceleration. Numerically we do it by means of $1 \mathrm{D}$ hybrid simulations, in which we introduce electrons as test-particles, aiming to see how the "hybrid" fields act on the individual electrons. The reduced spatial dimensionality allowed us to dramatically increase the number of macro-ions per cell and achieve the converged results for the velocity distributions of electrons. Our simulations show, that the electron-to-proton temperature ratio is a decreasing function of the shock Mach number. Furthermore, a saturation of the temperature ratio at higher Mach numbers is noticeable. This behavior is in agreement with the electron-ion temperature ratio observed in Balmer-dominated shocks [P. Ghavamian et al., Space Science Reviews, 178, 2013].
\end{abstract}

36th International Cosmic Ray Conference -ICRC2019-

July 24th - August 1st, 2019

Madison, WI, U.S.A.

${ }^{*}$ Speaker. 


\section{Introduction}

Collisionless shocks are ubiquitous in astrophysical environments and proven to be efficient particle accelerators, with supernova remnant (SNR) shocks being the most probable source of galactic cosmic rays [1]. The acceleration of charged particles at these shocks is well described by the theory of diffusive shock acceleration (DSA). While this mechanism is conceptually simple, but it is often difficult to implement under realistic shock conditions. In particular, the initial process of energetic particle injection into the DSA remains unsolved, with the electron injection being the most complicated problem. An important question is whether protons accelerated to high energies create sufficient wave turbulence required for the electron injection into the DSA. Furthermore, as the transition between the unshocked and the shocked medium is much shorter than the collisional mean free path, an equilibration of ion and electron temperatures might occur only on long timescales. Understanding the thermalization of electrons is important for the analysis of observed $\mathrm{X}$-ray spectra as well as for understanding the energy partitioning between the energetic CR and the thermal populations. Observations of Balmer dominated shocks have shown a dependence of the electron-ion temperature ratio on the shock velocity [2]. We investigate this dependence by introducing electrons as test-particles in hybrid simulations and study their thermalization in the proton driven turbulence.

\section{Model and Simulation Set-Up}

Following the evolution of a collisionless shock over many ion cyclotron times is not feasible by means of fully kinetic simulations with realistic electron-ion mass ratios. In this case hybrid simulations have been proven to be a valuable tool. In these simulations the electrons are treated as a charge neutralizing massless fluid,

$$
n_{e} m \frac{d \vec{v}_{e}}{d t}=0=-e n_{e}\left(\vec{E}+\frac{1}{c} \vec{v}_{e} \times \vec{B}\right)-\nabla p_{e}+e n_{e} \eta \vec{J}
$$

where $-e, m_{e}, n_{e}$, and $\vec{v}_{e}$ are the electron charge, mass, density and bulk velocity. The resistivity, $\eta$, and pressure, $p_{e}$, are assumed to be scalar and an adiabatic equation of state with adiabatic index $\gamma=5 / 3$ is used for the electron pressure. The ions are treated kinetically, and their motion is governed by the following non-relativistic equations:

$$
m_{i} \frac{d \vec{v}}{d t}=q_{i}\left(\vec{E}+\frac{1}{c} \vec{v} \times \vec{B}-\eta \vec{J}\right), \quad \frac{d \vec{x}}{d t}=\vec{v} .
$$

In our simulations the lengths are normalized to the ion inertial length, $c / \omega_{p}$, with the proton plasma frequency $\omega_{p}=\sqrt{4 \pi n_{0} e^{2} / m_{p}}$. Here $n_{0}$ denotes the upstream plasma density and $e$ and $m_{p}$ are the proton charge and mass, respectively. Time is measured in units of inverse proton gyrofrequency, $\omega_{c}^{-1}=\left(e B_{0} / m_{p} c\right)^{-1}$, and velocity in units of the Alfvén velocity, $v_{\mathrm{A}}=B_{0} / \sqrt{4 \pi n_{0} m_{p}}$. Here $B_{0}$ is the magnitude of the background magnetic field, which is set to be parallel to the shock normal in all simulations presented below. The simulation is initialized by sending a super-sonic and super-alfvénic hydrogen plasma against a reflecting wall, placed at $x=0$. A shock forms upon the interaction of the counter-propagating plasma streams and propagates in positive $x$-direction. 
While the electron scales are generally neglected in hybrid simulations we are nevertheless interested in the behavior of electrons in the fields generated by the ions. Hence we add a population of electrons as test-particles in our simulation. Due to the separation of scales we have introduced a sub-cycling routine in the code to properly resolve the trajectories of the test-particle electrons. The propagation of the electrons is performed in $N_{\text {cyc }}$ sub steps, reducing the effective time step for the electron propagation to $\Delta t_{e}=\Delta t / N_{\mathrm{cyc}}$. A linear interpolation between the fields known at the time-steps of the ion propagation $t=n \Delta t$ and $t=(n+1) \Delta t$ is used to obtain the fields at the sub steps. To reduce the numerical costs we use a slightly reduced electron-ion mass-ratio of $m_{e} / m_{p}=1 / 400$ and update the electron positions and velocities $N_{\text {cyc }}=20$ times during one propagation step of the ions, where we use $\Delta t=0.01 / v_{0}$. Note, that using the guiding center approximation or a gyro-kinetic treatment $[3,4]$ of the electrons would be also possible, however, starting from some energy, adiabaticity can stop working well for the electrons. The electrons are assumed to be initially in thermal equilibrium with the ions with $\beta_{e}=\beta_{p}=1$ and are injected at the right boundary starting from the beginning of the simulation with a velocity distribution according to a Maxwellian flux with drift velocity $v_{0}$ (for details see [5]). The simulations were performed with at least 1000 ions per cell and a cell size of $\Delta x=0.25 c / \omega_{p}$ to reduce the numerical noise in the fields, which can lead to an artificial heating of the electrons. In order to keep the high particle statistics the dimensionality of the simulations are reduced to $1 \mathrm{D} 3 \mathrm{~V}$, only one spatial dimension $(x$ in our convention), but all components of the velocity and fields are included.

\section{Results}

We have performed hybrid simulations for different initial upstream flow velocities, i.e., different shock Mach numbers and followed the evolution of the shock for several hundred ion cyclotron times. In the following we present results of the simulations investigating the behavior of electron test-particles moving in the turbulent fields created by the main ion component.

The left panel in Fig. 1 shows the ion density and the components of the magnetic field for a simulation run with $v_{0}=10 v_{\mathrm{A}}$ at $t=300 \omega_{c}^{-1}$. It can be seen that the ion density increases upon shock crossing. Circularly polarized Alfvén waves are excited by the streaming protons. As the magnetic field is frozen into the plasma these waves are advected downstream and compressed, leading to large amplitudes of the magnetic field behind the shock front. The right panel of Fig. 1 shows the evolution of the spatially dependent $B_{y}$ with time. The propagation of the shock to the right as well as the compression of the magnetic field is clearly visible.

Detailed information about the accelerated particles and the temperature can be extracted from the particle phase spaces. Figure 2 shows the distribution of protons (top) on the $\left(x, v_{x}\right)$ plane and electron distribution (bottom) on the $\left(x, v_{\|}\right)$plane at $t=400 \omega_{c}^{-1}$. Here $v_{\|}$is the electron velocity component parallel to the local magnetic field. In the proton phase space accelerated particles are visible in the up- and downstream. A large increase in the proton temperature can be inferred from the width of the proton distribution. For the test-particle electrons the initial Maxwellian flux in the far upstream widens in the precursor (between $x=1000 c / \omega_{p}$ and $x=2000 c / \omega_{p}$ ), indicating an increase in temperature. Furthermore, a population of counter-propagating particles with positive $v_{\|}$is present in the upstream. This indicates a reflection due to magnetic mirroring near the shock transition. The inset shows a region close to the shock transition, where this effect becomes visible. 

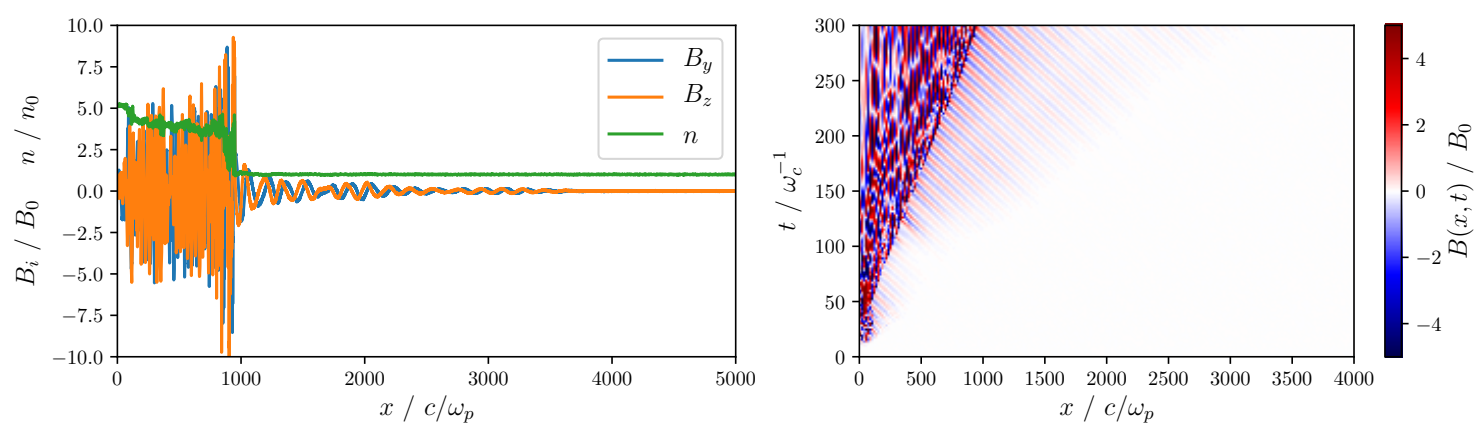

Figure 1: (left) Ion density $n_{i}$ and the components of the magnetic field $B_{y}, B_{z}$ for a simulation with upstream flow velocity $v_{0}=10 v_{\mathrm{A}}$ at $t=300 \omega_{c}^{-1}$. (right) $B_{y}$-component of the magnetic field as function of spatial coordinate and time.

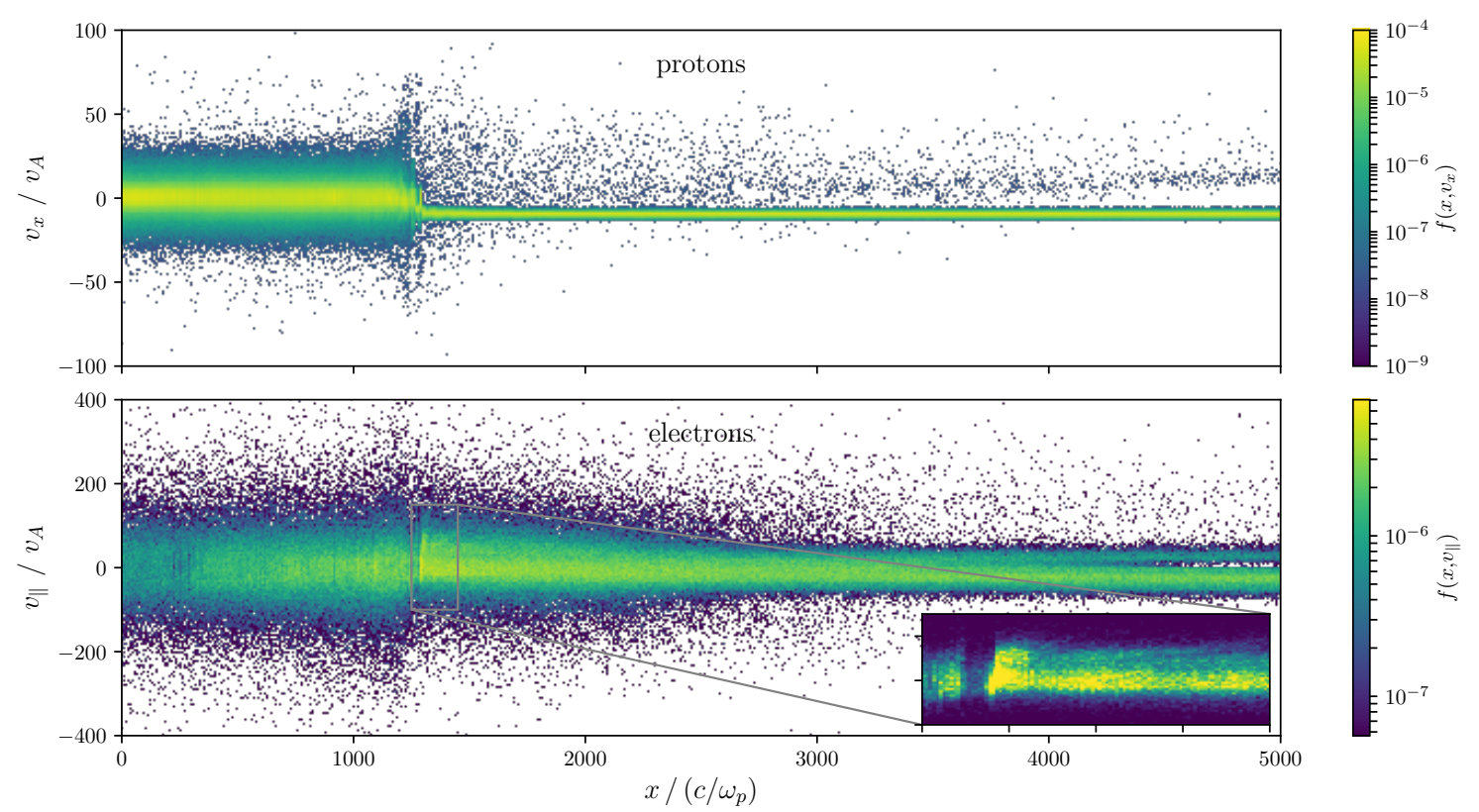

Figure 2: Phase space $f_{p}\left(x, v_{x}\right)$ of protons (top) and $f_{e}\left(x, v_{\|}\right)$of test-particle electrons (bottom) at $t=$ $400 \omega_{c}^{-1}$. The inset shows $f_{e}\left(x, v_{\|}\right)$close to the shock transition on a linear scale.

Upon shock crossing the width of the distribution in velocity changes only slightly, hence only a minor increase in temperature is expected.

To further investigate the reflection of electrons at the shock, we have traced some test-particles, which have been reflected in the time interval $275 \omega_{c}^{-1}<t<300 \omega_{c}^{-1}$, Fig. 3. The background shows the magnetic field $B(x, t)$. The line color denotes the energy in terms of the electrons initial energy

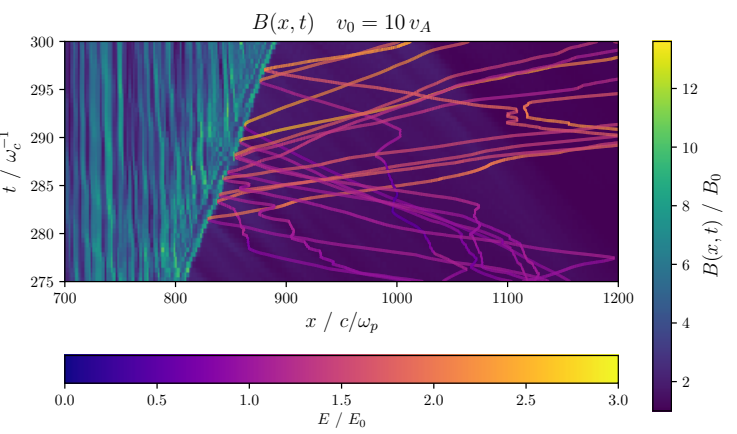

Figure 3: Trajectories of reflected electrons in the magnetic field $|B(x, t)|$. The line color denotes the energy of the test-particle electrons. 
test-particles gain energy upon reflection. This can also be seen from the phase space plot, Fig. 2, where particles with large positive $v_{\|}$are present. This process might be important for the injection of electrons into the DSA, however, the downstream energy spectrum of the test-particle electrons does not show a clear power-law tail.

The spatially dependent temperature profiles of protons (green), test-particle electrons (orange) and electron fluid (blue) are plotted in Fig. 4 for a simulation with upstream flow velocity $v_{0}=$ $10 v_{\mathrm{A}}$. The temperature of the test-particle electrons is $\sim 2$ times larger than the fluid temperature and a gradual increase of the temperature of the test-particles is clearly visible in the precursor.

By performing simulations for a range of initial flow velocities, $v_{0}$ (i.e., different shock velocities, $v_{\text {sh }}$ ) and calculating the downstream temperatures, we obtained the dependence of the electron-to-proton temperature ratio on the shock Mach number, $M_{A}=v_{\mathrm{sh}} / v_{\mathrm{A}}$. The results, presented in Fig. 5 by blue diamonds, clearly show that $T_{e} / T_{p}$ is a decreasing function of the shock Mach number. Additionally, we show on the same graph the temperature ratios determined from observations of Balmer-

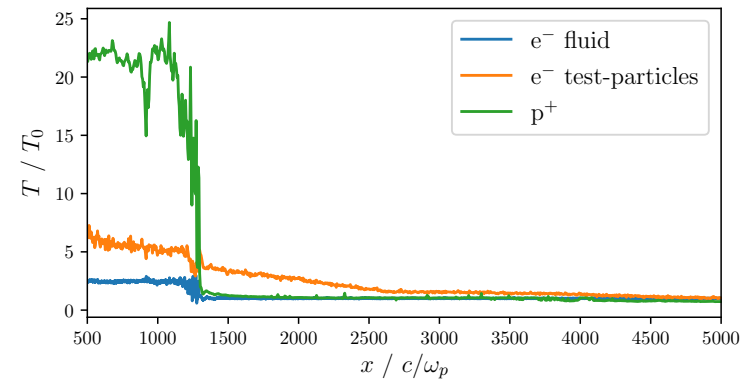

Figure 4: Spatial dependence of the temperature for the electron fluid, test-particle electrons, and protons at $t=400 \omega_{c}^{-1}$ for a simulation with $v_{0}=10 v_{\mathrm{A}}$. dominated shocks (orange) as function of the shock velocity $v_{\text {sh. }}$. The data is taken from $[2,6,7]$, where the authors extracted the information about the shock velocity and the temperature ratio from the width of the broad- and narrow components of the $\mathrm{H} \alpha$ line profile (see, e.g., Fig. 1 in [2]) ${ }^{1}$.

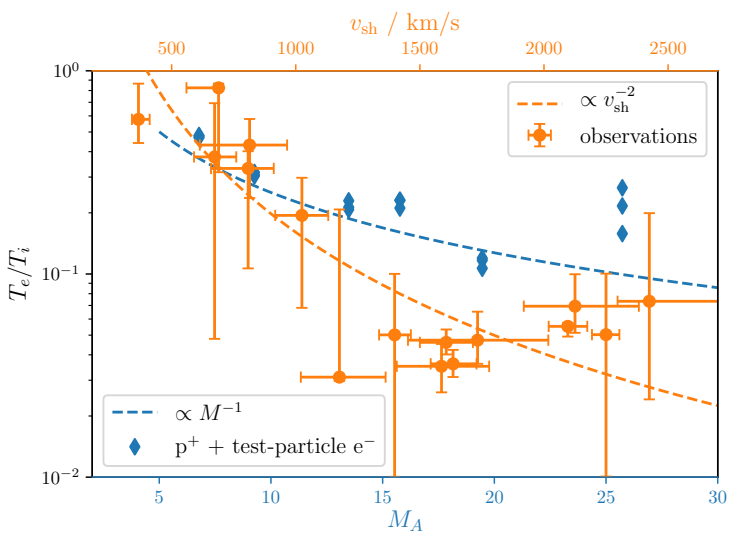

Figure 5: Electron to proton temperature ratio extracted from the simulations (blue diamonds) compared to the temperature ratio determined from observations of Balmer-dominated shocks (orange).
The observational data in Fig. 5 (orange) show that the temperature ratio $T_{e} / T_{p}$ decreases with increasing shock velocity and at high shock velocities it seems to saturate. A dependence of $T_{e} / T_{i} \sim v_{\mathrm{sh}}^{-2}$ was found to fit best to the observational data [2] (dashed orange line in Fig. 5). Instead, a function $T_{e} / T_{i} \sim M^{-1}$ is fitted to our simulation results (dashed blue line in Fig. 5). This dependence was found earlier for the terrestrial bow shock and interplanetary shocks [8]. However, our simulation only treat the initial (injection) phase of CR acceleration and all particles are sub relativistic. Hence, the scaling law might be the same as for interplanetary shocks. Note that a relatively

\footnotetext{
${ }^{1}$ Note, that since the environmental conditions of the SNR shocks could not be determined directly the shock Mach numbers were not known.
} 
high Alfvén velocity of $v_{A}=90 \mathrm{~km} / \mathrm{s}$ has to be assumed to directly compare the measurements with the simulation data. This is about 4 times larger than the velocity one would expect when using the standard parameters of the ISM (for $B=3 \mu \mathrm{G}$ and $n=0.1 \mathrm{~cm}^{-3}$ the Alfvén velocity equals $v_{A} \simeq 20 \mathrm{~km} / \mathrm{s}$ ). However, the exact conditions of the SNR environment are not known and one might have to consider the Alfvén velocity in the field ahead of the shock [2] where the magnetic field is higher, $\Delta B / B=4-10$.

\section{Discussion}

Our simulations as well as the observational results from Balmer-dominated shocks indicate that an equilibration of electron and ion temperatures seems only to occur at slow shocks, while for higher shock velocities a decreasing electron-ion temperature ratio is observed. Furthermore, the observations also show a saturation or even an upturn of the temperature ratio for high shock velocities. This saturation is on a level which is higher than the mass proportional heating, expected from the Rankine-Hugoniot jump conditions,

$$
k T_{e, i}=\frac{3}{16} m_{e, i} v_{\mathrm{sh}}^{2}
$$

The observed scaling is also supported by theoretical predictions: Vink et al. [9] have shown that a dependence of $T_{e} / T_{i} \sim M_{s}^{-2}$, where $M_{s}=v_{\mathrm{sh}} / c_{s}$ is the sonic Mach number, can be obtained by solving the Rankine-Hugoniot jump conditions with the assumption that the enthalpy flux is conserved for each particle species separately. Under certain assumptions this yields a $\sim M_{s}^{-2}$ behavior for shocks with Mach numbers in the range

$$
\sqrt{\frac{2}{\gamma-1} \frac{\mu}{m_{i}} \frac{r^{2}}{r^{2}-1}}<M_{s}<\sqrt{\frac{2}{\gamma-1} \frac{\mu}{m_{e}} \frac{r^{2}}{r^{2}-1}}
$$

with $\mu=\left(m_{i}+m_{e}\right) / 2$ being the average mass and $r$ the compression ratio. Using the increased electron mass from our simulations this translates to $1.4<M_{A}<23$. Furthermore, in case of efficient $\mathrm{CR}$ acceleration, i.e., when considering the $\mathrm{CR}$ pressure in the precursor, Vink et al. also predict a higher level of saturation of the temperature ratio towards high $M_{s}$. The predicted range for the $M_{s}^{-2}$ scaling, with the dependence on $m_{e}$ and $m_{i}$ also disfavors fully kinetic simulations, where regularly the reduced mass-ratios of $m_{p} / m_{e}=64-100$ are used [10]. In this case, the scaling is expected to occur only in a limited range of Mach numbers.

While we have not identified the mechanism responsible for the heating of the test-particles, different models have been proposed for the heating of electrons in front of SNR shocks. All these models have to rely on numerical simulations since the particle distributions in SNR shock cannot be measured in situ. When focusing on non-relativistic collisionless shocks, mainly two scenarios have been considered: heating due to lower hybrid waves in the precursor [11] or a mechanism based on counterstreaming instabilities in front of the shock [12]. Both mechanisms work well for perpendicular shocks, however, it has been argued that due to the amplification of the magnetic field ahead of the shock, the perpendicular component might be large enough for the models to be applicable $[13,14]$ also for quasi-parallel shocks. 


\section{Acknowledgments}

The research was supported by DFG grant TL 2479/2-1 and by NASA ATP-program within grant 80NSSC17K0255. The authors acknowledge the North-German Supercomputing Alliance (HLRN) for providing the computational resources for the simulations.

\section{References}

[1] T. K. Gaisser, Cosmic Rays and Particle Physics. Jan. 1991.

[2] P. Ghavamian, S. J. Schwartz, J. Mitchell, A. Masters, and J. M. Laming, "Electron-ion temperature equilibration in collisionless shocks: The supernova remnant-solar wind connection," Space Science Reviews, vol. 178, pp. 633-663, Oct 2013.

[3] E. A. Frieman and L. Chen, "Nonlinear gyrokinetic equations for low-frequency electromagnetic waves in general plasma equilibria," The Physics of Fluids, vol. 25, no. 3, pp. 502-508, 1982.

[4] R. G. Littlejohn, "Variational principles of guiding centre motion," Journal of Plasma Physics, vol. 29, no. 1, pp. 111-125, 1983.

[5] K. Cartwright, J. Verboncoeur, and C. Birdsall, "Loading and injection of maxwellian distributions in particle simulations," Journal of Computational Physics, vol. 162, no. 2, pp. 483 - 513, 2000.

[6] P. Ghavamian, J. M. Laming, and C. E. Rakowski, "A physical relationship between electron-proton temperature equilibration and mach number in fast collisionless shocks," The Astrophysical Journal Letters, vol. 654, no. 1, p. L69, 2007.

[7] M. van Adelsberg, K. Heng, R. McCray, and J. C. Raymond, "Spatial structure and collisionless electron heating in balmer-dominated shocks," The Astrophysical Journal, vol. 689, no. 2, p. 1089, 2008.

[8] S. J. Schwartz, M. F. Thomsen, S. J. Bame, and J. Stansberry, "Electron heating and the potential jump across fast mode shocks," Journal of Geophysical Research: Space Physics, vol. 93, no. A11, pp. 12923-12931.

[9] Vink, Jacco, Broersen, Sjors, Bykov, Andrei, and Gabici, Stefano, "On the electron-ion temperature ratio established by collisionless shocks," A\&A, vol. 579, p. A13, 2015.

[10] J. Park, D. Caprioli, and A. Spitkovsky, "Simultaneous acceleration of protons and electrons at nonrelativistic quasiparallel collisionless shocks," Phys. Rev. Lett., vol. 114, p. 085003 , Feb 2015.

[11] J. M. Laming, "Electron heating at snr collisionless shocks," The Astrophysical Journal Supplement Series, vol. 127, no. 2, p. 409, 2000.

[12] P. J. Cargill and K. Papadopoulos, "A mechanism for strong shock electron heating in supernova remnants,” The Astrophysical Journal Letters, vol. 329, pp. L29-L32, June 1988.

[13] C. E. Rakowski, J. M. Laming, and P. Ghavamian, "The heating of thermal electrons in fast collisionless shocks: The integral role of cosmic rays," The Astrophysical Journal, vol. 684, no. 1, p. 348, 2008.

[14] M. A. Malkov, A. A. Galeev, and H. J. Völk, "Electrostatic Shocks Driven by Accelerated Electrons," International Cosmic Ray Conference, vol. 3, p. 273, 1995. 